Theatre Research in Canada

Recherches théâtrales au Canada

\title{
Présentation: Pédagogie et édition
}

\section{Kim Solga}

Volume 39, Number 1, 2018

URI: https://id.erudit.org/iderudit/1055464ar

DOI: https://doi.org/10.7202/1055464ar

See table of contents

Publisher(s)

Graduate Centre for the Study of Drama, University of Toronto

ISSN

1196-1198 (print)

1913-9101 (digital)

Explore this journal

Cite this document

Solga, K. (2018). Présentation: Pédagogie et édition. Theatre Research in Canada / Recherches théâtrales au Canada, 39(1). https://doi.org/10.7202/1055464ar

All Rights Reserved (c) Theatre Research in Canada / Recherches théâtrales au Canada, 2018
This document is protected by copyright law. Use of the services of Érudit (including reproduction) is subject to its terms and conditions, which can be viewed online.

https://apropos.erudit.org/en/users/policy-on-use/
This article is disseminated and preserved by Érudit.

Érudit is a non-profit inter-university consortium of the Université de Montréal, Université Laval, and the Université du Québec à Montréal. Its mission is to promote and disseminate research.

https://www.erudit.org/en/ 


\section{Pédagogie et édition}

KIM SOLGA

J'ai vécu ma première expérience formatrice du travail d'édition bien avant de devenir moimême éditrice. C'était au début des années 2000 , et suivant le conseil d'un de mes professeurs, je venais de transformer en article une dissertation sur Mère courage et ses enfants qui m'avait valu une note particulièrement bonne. Mes amis et mes mentors m'ayant conseillé de viser haut, j'ai envoyé l'article à Theatre fournal (après l'avoir révisé, bien sûr, mais en négligeant, comme j'allais bientôt le découvrir, d'en faire un travail d'édition). Quelques semaines plus tard, j'ai reçu une jolie lettre de refus du rédacteur en chef de l'époque, David Román. Ce dernier m'a écrit des choses très gentilles au sujet de mon texte, mais la conclusion de sa missive a tout de même eu un effet dévastateur sur moi : il n'était pas prêt à le soumettre à un examen par des pairs.

J'étais abattue. Ce texte m'avait valu tant de louanges de mon professeur sur ses forces théoriques et son originalité. Comment M. Román ne pouvait-il pas être du même avis? Étaitce parce que même mes meilleurs efforts ne suffiraient tout simplement pas? J'ai versé quelques larmes, j'ai écrit à mon meilleur ami pour me vider le cœur (ce dernier, encourageant comme toujours, m'a appuyé en se fâchant lui aussi), puis, après un temps de deuil, j'ai contacté Joanne Tompkins.

Joanne (avec Ric Knowles et Bill Worthen) était ma superviseure (et l'une des rédactrices en chef) à Modern Drama, où je travaillais à l'époque comme adjointe à la rédaction. C'est à moi que revenait la responsabilité de vérifier les citations - une tâche d'assistanat dont peu de gens connaissent l'existence, mais qui est absolument essentielle dans tout organe de publication - , et grâce à ce travail (et au soutien généreux de Bill, de Joanne et de Ric), j'ai appris, lentement mais sûrement, à quoi ressemble un bon article au moment d'arriver dans la boîte de réception d'un éditeur, comment évolue ce texte au fil d'un travail d'édition minutieux, et surtout, à quel point il peut inévitablement y rester des petites erreurs de formatage et de citation, même à la toute fin du processus. (La plus grande leçon que j’ai tirée de mon emploi chez Modern Drama est la suivante : vérifiez toujours vos citations avant d'envoyer votre texte!)

Comme Joanne était ma mentore, je savais que je pouvais compter sur elle pour m'aider à traverser ce moment de difficulté rédactionnelle. Je lui ai demandé de lire mon article (dans sa version refusée) et de me livrer ses impressions; elle a gentiment accepté de le faire. Ce qu'elle m'a dit m'a marqué à vie. Non seulement je n'ai jamais oublié ses conseils, mais j'en fais un point d'honneur de les transmettre à tous mes étudiants de deuxième et troisième cycles, de même qu'à tout auteur en émergence (jeune ou moins jeune) avec qui je fais un travail d'ordre éditorial.

Premièrement, un travail universitaire ne peut pas être publié tel quel dans une revue : c'est un tout autre type de document (tout comme une demande subvention n'est pas un travail universitaire ou un article) régi par un tout autre type de convention (et même, parfois, une tout autre structure). Deuxièmement, on n'a pas à s'éterniser sur les écrits déjà parus sur 
le sujet ; on gagne plutôt à présenter notre hypothèse d'entrée de jeu et à dire tout de suite au lecteur pourquoi on doit s'intéresser à la question, pour ensuite faire marche arrière et fournir des éléments de contexte peu connus des lecteurs interpellés par notre postulat. Troisièmement, on gagne à bien " baliser » son texte, en montrant constamment au lecteur comment se déploie l'argumentation et en lui expliquant comment chacune des composantes de notre article se lie au texte dans son ensemble. Enfin, il va sans dire que certains articles, en voulant tout faire, sont voués à l'échec ; on pourrait les appeler les 2-en-I. Les deux idées vraiment intéressantes que présente un tel article sont certainement associées, mais quel est leur rapport ici, dans ce texte? Est-ce qu'il y a vraiment un lien entre elles? Y aurait-il un moyen de modifier légèrement la structure du texte afin de rendre ce lien plus clair, tant pour l'auteur que pour ses lecteurs? (La structure d'un texte, m'a dit Joanne, équivaut à $50 \%$ de son contenu : sans l'appui d'une bonne structure, même les idées les plus brillantes se perdent. C'est ma remarque préférée à écrire sur les copies de mes étudiants au bac.)

Les conseils et l'appui qui m'ont été offerts par Joanne à la suite de mes débuts manqués au Theatre fournal ont non seulement mené à une première publication évaluée par les pairs, mais ont aussi servi à mes débuts comme éditrice. Toutes ces années plus tard, après bien des projets d'édition et de nombreuses collaborations avec des revues, je constate que j'ai appris plein de choses sur mon propre processus d'écriture en lisant et en révisant les textes des autres. J'ai également eu l'occasion de voir le fruit des conseils de Joanne, que j'ai pu transmettre à d'innombrables étudiants et collègues alors qu'ils travaillaient au développement de leur écriture. Quand je me demande pourquoi j'ai choisi de me consacrer à ce point au travail d'édition, pourquoi je dis rarement non quand un collègue me demande de lire un de ses textes, je pense à la grande satisfaction que m'apporte ce processus d'enseignement et d'apprentissage. Je pense aussi, avec fierté, aux nombreux magnifiques projets de recherche auxquels se sont consacrées ces personnes que j'ai pu aider un peu, ou beaucoup, à mettre leurs idées sur papier.

Ce numéro de TRIC/RTAC célèbre de maintes façons le travail des mentors et la puissance intarissable de nos pédagogies. Il commence par une réflexion marquante par Colleen Kim Daniher sur une expérience d'enseignement qui traverse les frontières raciales et ethniques. Dans une contribution intitulée "On Teaching Kim's Convenience in the Asian American Theatre and Performance Studies Classroom ", Daniher part d'une expérience qu'elle a elle-même vécue alors qu'elle était postdoctorante à l'université Brown pour réfléchir aux histoires entretissées du militantisme des Canadiens et des Américains d'origine asiatique, au " mythe du modèle de la minorité » et aux relations entre les communautés noires et asiatiques d'Amérique du Nord. Dans sa contribution, elle propose une méthodologie interdisciplinaire et transfrontalière pour enseigner les œuvres de créateurs canadiens d'origine asiatique dans un contexte transnational et lance un puissant appel à l'action pour la mise au point d'un champ d'études consacré au théâtre canado-asiatique. (Soit dit en passant, je suis particulièrement fière de pouvoir publier cet article ici puisque Daniher était l'une de mes premières étudiantes en théâtre à l'Université Western en 2005.)

À la suite de l'article de Daniher se trouve une contribution par Monique Hamel intitulée "La question du "réel et son double" dans les créations théâtrales au secondaire ", où il est question des effets du " double ", une fonction centrale à la création théâtrale, sur les explorations créatives et les processus d'apprentissage d'élèves du secondaire. Hamel s'interroge 


\section{PRÉSENTATION}

comme suit : «Comment la question du double se dégage-t-elle du travail de création et en quoi est-il significatif dans le cheminement de l'élève d'un point de vue éducatif, artistique, ainsi que du point de vue du spectateur? ». Pour trouver des éléments de réponse, elle se sert de trois créations théâtrales et d'une série d'entretiens traçant l'expérience vécue par six élèves participants devenus adultes. Si Daniher propose une nouvelle façon de penser les moyens dont nous nous servons pour structurer et communiquer les différences complexes dans nos salles de classe, Hamel explore comment le fait de vivre à l'intérieur des structures les plus élémentaires de la différence occasionnée par le théâtre peut aider les élèves à entamer un processus d'apprentissage sur eux-mêmes et sur les autres qui les suivra tout au long de leur vie.

Kelsey Jacobson reprend le fil du « double » et celui du « réel » que tend Hamel pour partir sur la trace du Trinity Pageant, un spectacle présenté par la compagnie Rising Tide Theatre dans diverses collectivités isolées de Terre-Neuve. Dans "Through the Fictive to the Real(ish): Affective Time and the Representation of "Real Newfoundland" in Rising Tide Theatre's Trinity Pageant ", Jacobson s'intéresse au théâtre localisé, aux théories du temps performatif et aux pratiques de la reconstitution historique en s'interrogeant sur la nature $\mathrm{du}$ «Terre-Neuve véritable » que cherche à dépeindre le Trinity Pageant. Hommage affectueux à l'histoire orale d'une collectivité et critique riche des divers moyens par lesquels les cultures de la performance sont souvent à la merci de la logique économique dans les collectivités rurales ou semi-rurales en cette ère post-industrielle, l'article de Jacobson demande si la "vérité " (approximative) du Terre-Neuve que propose le Trinity Pageant, semi-exacte et reposant largement sur l'affectif, n'est pas, en fin de compte, plus réalité que fiction-d'autant plus s'il s'agit d'une bouée qui permet à des collectivités comme celle de Trinity de survivre au XXI ${ }^{\mathrm{e}}$ siècle dans le contexte d'un Canada en proie à la mondialisation et au néo-libéralisme.

Jacobson est une doctorante à l'Université de Toronto, et elle signe une deuxième contribution à ce numéro avec «Accuracy and Ethics, Feelings and Failures: Youth Experimenting with Documentary Practices of Performing Reality ", coécrite avec son collègue Scott Mealey, lui aussi doctorant à l'Université de Toronto, et la professeure Kathleen Gallagher, avec qui Jacobson et Mealey ont collaboré à un projet de recherche subventionné par le CRSH. Modèle de mentorat savant et dialogue fascinant sur la recherche, ce dernier article explore l'utilisation des pratiques associées au théâtre documentaire et à l'histoire orale dans trois salles de classe conceptuelles au Canada et à Taïwan. Constatant le besoin des jeunes artistes de théâtre de «bien » transmettre leurs histoires sur scène, Gallagher, Jacobson et Mealey se demandent comment l'enchâssement d'une éthique de l'échec dans le processus de révélation de la vérité peut venir appuyer un engagement plus rigoureux envers les enjeux sociopolitiques complexes auxquels les jeunes sont confrontés.

Ce numéro se conclut par un Forum plus volumineux qu'à l'ordinaire, où deux autres exemples de pédagogie radicale vous sont proposés par nos contributeurs. Le premier texte consiste en un échange présenté par Glen Nichols sur les pouvoirs souvent cachés des arts libéraux - et notamment des études théâtrales - que l'on enseigne dans les universités canadiennes. Il se fonde sur de longues années d'échanges en personne et en ligne dans le cadre de colloques de l'ACRT. Ensemble, Claire Carolan, Justin Bloom, Moira Day, Shelley Scott et James McKinnon font un plaidoyer puissant sur la valeur de l'enseignement du théâtre et 
de la performance comme "paradigme critique mobile " (l'expression est empruntée à Freeman et Gallagher, In Defence of Theatre, 2016). En cours de route, les intervenants offrent des exemples d'innovation pédagogique exceptionnelle dont tout enseignant, chevronné ou novice, voudra sans doute s'inspirer. Dans la deuxième contribution au Forum - qui occupera la place d'honneur dans ce numéro en ligne-Ric Knowles, Natalie Alvarez et l'équipe de la troupe Modern Times Theatre Company partagent avec nous une version abrégée d'un rapport découlant de l'atelier et du symposium « Post-Marginal » qu'ils ont organisé en avril 20I7. Dans leur texte, ils s'interrogent sur les moyens à emprunter pour aller au-delà des belles paroles sur la valeur de la diversité sur scène et inscrire la diversité au cœur de nos pratiques associées aux répétitions, à la critique, à la mise en scène et au visionnement du théâtre. Le document (ainsi que la galerie d'images et la série de liens virtuels qui l'accompagnent sur le Web) offre une fenêtre précieuse sur un événement révolutionnaire aux personnes qui n'ont pas pu y assister en personne l'an dernier et sert de rappel à celles et ceux d'entre nous qui ont eu l'occasion d'y être.

Ce numéro de TRIC/RTAC marque le début d'une nouvelle pratique pour la revue, celle d'exister principalement en ligne. À l'avenir, nos abonnés qui voudront absolument obtenir une copie papier de la revue devront la demander. Que vous la lisiez sur papier ou à l'écran, vous pourrez continuer de compter sur notre engagement à diffuser le fruit de recherches sur le théâtre et la performance au Canada. Grâce aux économies que nous permettra de réaliser cette nouvelle formule, nous serons en mesure de vous proposer du contenu additionnel sur notre site Web. J'espère qu'une nouvelle génération de pédagogues et d'apprenants, d'auteurs et d'éditeurs pourra continuer d'être interpellée à interagir avec TRIC/RTAC dans les années à venir. 